\title{
An Automated Fume Control System for Automobile
}

\author{
Manpreet Kaur \\ Academic \& Consultancy Division (ACSD), \\ Centre for Development of Advanced \\ Computing (C-DAC), Mohali, 160071, India.
}

\author{
Nikhil Panwar \\ Academic \& Consultancy Division (ACSD), Centre \\ for Development of Advanced Computing (C- \\ DAC), Mohali, 160071, India.
}

\begin{abstract}
Continuous innovations and modernization has greatly contributed to the development in automobile industry. Automobile has become a huge luxury for everyone in all the countries. All modern automobiles like cars are equipped with air conditioner and heater units. An air conditioner alters the properties of air (temperature and humidity) to more comfortable conditions in building, house or automobile. But despite having various advantages, there are few drawbacks as well. Earlier the external pollution was the main concern but nowadays due to use of air conditioner in the car the fumes like carbon monoxide, sulphur dioxide etc. enters inside the cabinet through the inlets of the AC if there is any leakage. Heater may also produce fumes inside the cabinet if running for long time and ventilation is not there. Fumes like Carbon monoxide is lackluster, unscented, weak and exceptionally lethal and some harmful effects of this gas are cerebral pain, tipsiness, shortcoming, queasiness, regurgitating, midsection agony, and disarray. So a prototype is designed which detects the presence of gas inside the cabinet then alert the user by an alert system and automatically open the window to let the fresh air come inside the vehicle. If the level of $\mathrm{CO}$ increases continuously then it will automatically send an alert message with location to the concerned person related to the victim through GSM and GPS.
\end{abstract}

\section{Keywords}

GSM, GPS, CO, MQ7, PIC

\section{INTRODUCTION}

Engine vehicles are the prime wellspring of transportation, as well as the prime wellspring of contamination. These engine vehicles add to more than $30 \%$ of the hazardous gasses that are discharged into the air. Open air contamination levels are the key concern, yet the nature of air inside the vehicle has significant influence. As the vehicle lodge is confined space, any suspended particulate, for example, dust, exhaust, smokes entering into it through either ventilation or through pipes of air conditioner or heater can bring about genuine health issues to the individual inside. These particulate matters can bring about issues which may vary from intense and unending impacts on human health. The health impacts range from minor disturbance of eyes and the upper respiratory framework to unending respiratory ailment, coronary illness, lung malignancy and even

demise [2]. Regardless of the fact that the vehicle is outfitted with the air lodge channels that are available inside the cabin in order to reduce the impact of the undesirable particulate, they can't be a feasible response for control it. Indeed despite the fact that the makers of different vehicles predominantly focus on these security precautionary measures, the lack of regard of the end clients or the drivers in regards to the state of the vehicle may bring about genuine harm to the vehicles as well as to the human lives. Such that even the minor eye aggravation can make the driver to lose his fixation out and about, this may prompt genuine miss-chances. Subsequently to defeat these sorts of issues there is a need to screen a few gases [2].

In this work the main focus was on carbon monoxide as it forms very easily and it enters through the ventilation pipes. Carbon monoxide gas is lackluster, unscented, dull and lethal in nature. It is formed by the partial burning of a mixture of fuels. If one inhales this gas, this proves to be fatal. The continuous exposure to $\mathrm{CO}$ even at lesser amount is toxic. Among all the gases the probability of entering carbon monoxide inside the cabinet is more and it is more dangerous because of its unscented, dull properties it cannot be detected and effects human body quickly. In this research work, my prime focus is to detect the level of $\mathrm{CO}$ fumes inside the car cabinet. Carbon monoxide harms if very small quantity of the gas is inhaled. When carbon monoxide is breath in, it gets into the circulation system and keeps red platelets away from conveying oxygen. Without oxygen, body tissue and cells bite the dust. Long haul impacts of carbon monoxide harming incorporate paralysis and damage to brain. Carbon monoxide is harmful when inhaled on the grounds that it uproots oxygen in the blood and denies the heart, cerebrum, and other imperative organs of oxygen. A lot of $\mathrm{CO}$ inhaled makes you lose cognizance and choke within minutes. Carbon monoxide side effects are influenza, sustenance harming, viral diseases and basically tiredness [1]. That is the reasons for individuals often confuse this exceptionally perilous harming for something else. Introductory manifestations of CO harming may incorporate migraine, weariness, wooziness, tiredness, or queasiness. Amid delayed or high exposures, manifestations may compound and incorporate regurgitating, perplexity, and fall notwithstanding loss of cognizance and muscle shortcoming. In this paper designs a prototype for fume $\mathrm{CO}$ and oxygen level detection inside the vehicle cabinet. A microcontroller PIC 18F452 and sensor network of MQ-7 sensors are use to develop the sensing unit. If fume reaches the threshold level, sensing unit detects, an alarm is generated and automatically opens the window to let the fresh air come inside the vehicle. If fume level rises continuously then automatically sends an alert message with present location to the concerned person related to the victim through GSM and GPS module.

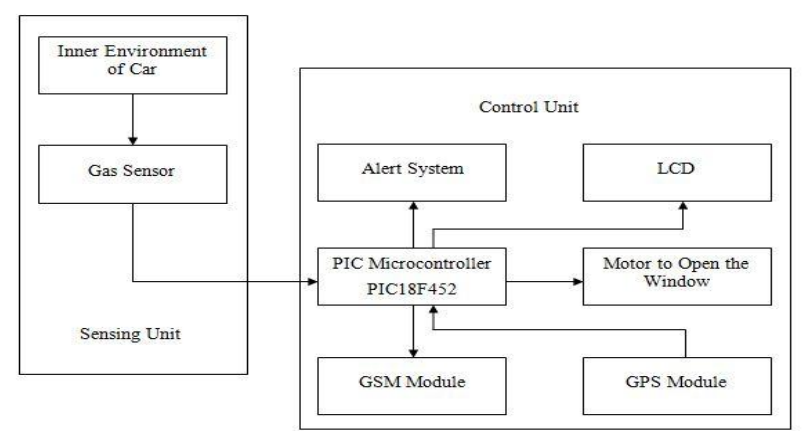

Fig 1: Block diagram of prototype system 


\section{PREVIOUS WORK}

A device is developed by Boris Raic et al. to minimize overheating within a vehicle which is parked in the hot summer sun and accordingly to make the entry into the vehicle more agreeable, microcontroller oversaw module for programmed ventilation of vehicle inside is made [3]. The module is actualized utilizing a microcontroller as a focal legitimate unit and a progression of sensors which give adequate information to guarantee practical, additionally proficient, solid and safe ventilation. The ventilation procedure is performed by opening vehicle windows somewhat, which empowers air to circle. Microcontroller controls the position of the windows self-sufficiently and freely of the driver's vicinity, taking after predefined calculation that uses sensors information got from the vehicle's environment. Other than temperature, the most imperative variables to guarantee quality execution of ventilation are distinguished developments around the vehicle, the vicinity of precipitation and other.

\section{HARDWARE DESCRIPTION}

\subsection{PIC microcontroller}

Controller is the main component as it works as the mind for the whole system designed. Microcontroller has crucial role in any control unit.

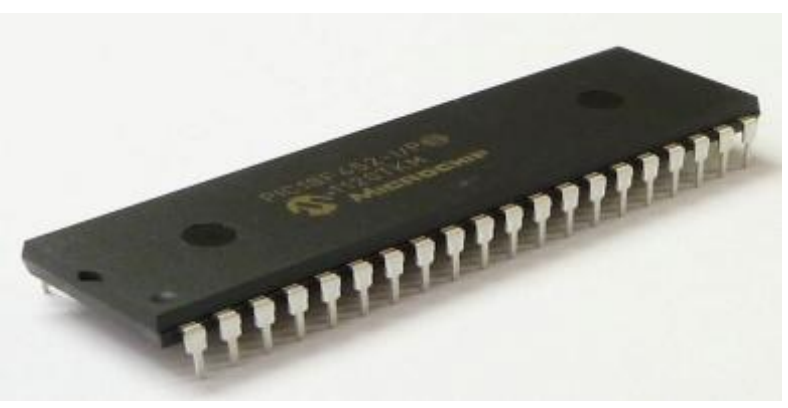

Fig 2: PIC18F452

The controller used in developing this device is PIC microcontroller PIC18F452. It is having high performance and flash programmable memory. The flash memory can be reprogrammed many times with the help of a program burner kit and the supporting software. It is having $32 \mathrm{~Kb}$ of program memory, 1536 bytes of RAM and 256 bytes of EEPROM. It has 3 interrupt pins externally. It runs up to $40 \mathrm{MHz}$ of clock speed. In this research nh work $8 \mathrm{MHz}$ crystal oscillator has been used for clock frequency. To connect crystal two capacitor of $22 \mathrm{pF}$ are used. MCLR pin is an active low pin of microcontroller. Microcontroller will reset itself and terminates all its activities whenever this pin gets an active low pulse.

\subsection{Gas sensor}

The change in the environment inside the car is to be detected using the gas sensors network. The changes occur in the environment due to presence of a particular gas are detected by a particular sensor. Some of the gases cannot be detected manually. The requirement in this project work is to detect and measure the carbon monoxide (CO) gas. So, there is need of $\mathrm{CO}$ detecting gas sensor. Among many other semiconductor sensors MQ-7 sensor has been selected to detect carbon monoxide as explained in following section. Carbon monoxide cannot be detected manually because of its colorless, odorless and non-irritating properties so a sensor is required to detect this gas. MQ-7 sensor is used in this project work to detect the level of $\mathrm{CO}$ inside the cabinet because it has quick response and short reactive time in detecting $\mathrm{CO}$ [6].
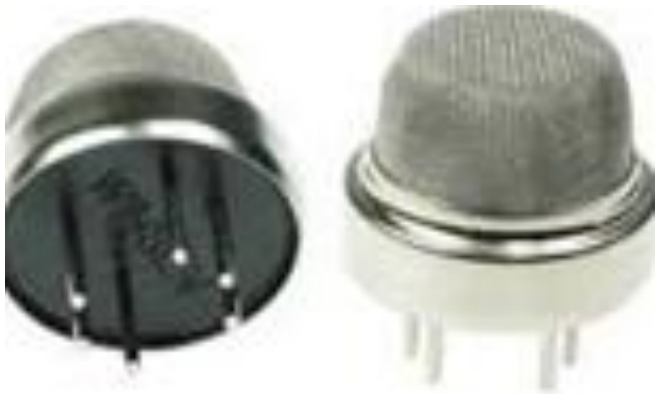

Fig 3: MQ-7 sensor

Table 1: Specifications of MQ-7 Sensor

\begin{tabular}{|l|l|}
\hline Parameter Name & Conditions \\
\hline Circuit voltage & $5 \mathrm{~V} \pm 0.1$ \\
\hline Heating voltage (HIGH) & $5 \mathrm{~V} \pm 0.1$ \\
\hline Heating voltage (LOW) & $1.4 \mathrm{~V} \pm 0.1$ \\
\hline Load resistance & Adjustable \\
\hline Heating resistance & $33 \Omega \pm 5 \%$ \\
\hline Heating time (HIGH) & $60 \pm 1$ seconds \\
\hline Heating time (LOW) & $90 \pm 1$ seconds \\
\hline Heating consumption & About 350mW \\
\hline Detecting range & $20 \mathrm{ppm}-2000 \mathrm{ppm}$ \\
\hline
\end{tabular}

\subsection{LCD display}

LCD display is used to continuously show the monitored level of the fume inside the cabinet. It shows the continuous volumeof carbon monoxide gas inside the cabinet. A Liquid Crystal Display (LCD) is a thin, flat electronic visual display that uses the light modulating properties of liquid crystals (LCs). It is a low cost, low power device capable of displaying text and images. LCDs are extremely common in embedded systems. They are usually more compact, lightweight, portable, less expensive, more reliable, and easier on the eyes. Only alphanumeric characters are displayed on LCD for which ASCII numbers are sent on data pins of LCD by the microcontroller [4].

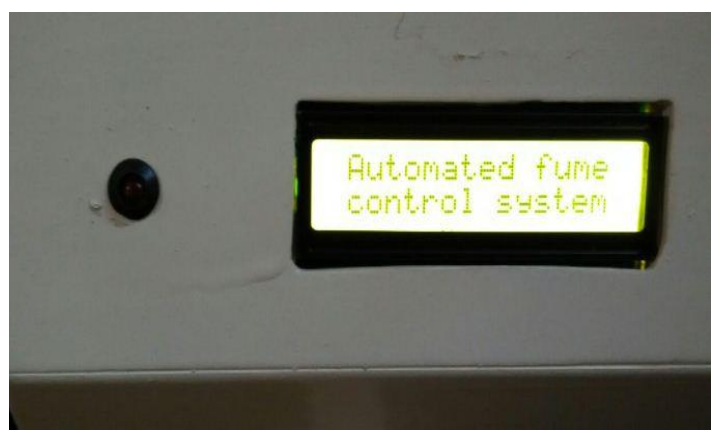

Fig 4: LCD Display on prototype 


\subsection{DC motor}

DC motor is an electromechanical device that converts DC electrical energy into mechanical energy. The principle on which the DC motor works is that when a conductor carrying a current is positioned in a magnetic field, a torque is experienced by the conductor and it tends to move. This action is recognized as monitoring action. To reverse the direction of the DC motor, the current in the conductor is also reversed. Thus the principle of DC motor can be summarized as the interaction of both the electric and the magnetic field which produces a mechanical force. When the level of the fume has increased up to the danger level then the window of the car is open using the motor.

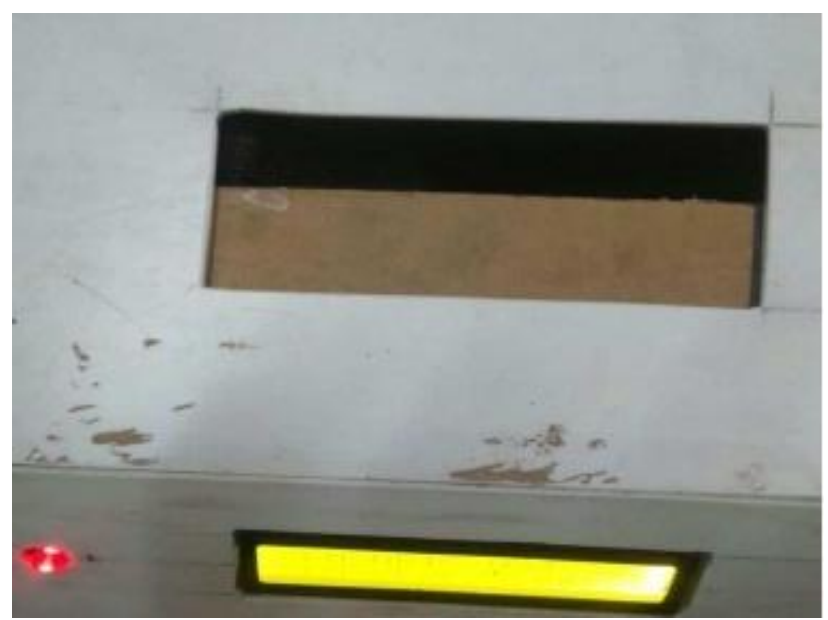

Fig 5: Window opens when limit exceeds than typical level

\subsection{GSM module}

GSM module is used if the level of fume inside the car increases up to the danger level and the person sitting inside the car. Then a message send to the person related to the victim about the fume status and location of car.

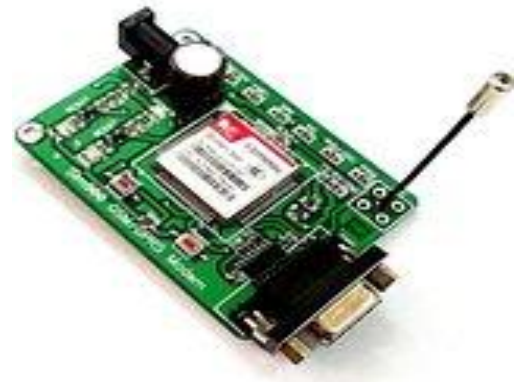

Fig 6: GSM module

GSM Modem used for massage transition is SIM900 Quad band. It works on frequency $850 \mathrm{MHZ}$ or $900 \mathrm{MHZ}$ and $1800 \mathrm{MHZ}$ or 1900MHZ.It is very compact in size and operative voltage required is $12 \mathrm{v}$ DC. The baud rate can be configured from 9600-115200 bps through AT (Attention) commands. It is used for massage transfer and data transfer applications. It is easily interface with microcontroller or to computer by using UART, serial communication [8].

\subsection{GPS module}

When the danger level has increased and a message is send to the someone related to the person sitting inside using GSM module then in that message the location of the vehicle is also send using the GPS module so the help can be send to that location and the safety measures can be provided to him. Satellite navigation technology is used in the GPS module. The latitude and longitude positions are located by the GPS receiver. GPS provides the information in a string format. In this research work The U-blox PAM-7Q series GPS module is used for navigation aid. It has $18 * 18 \mathrm{~mm}$ patch antenna which provides RHCP polarization and accuracy of $2.5 \mathrm{~m}$ CEP, SBAS $2.0 \mathrm{~m}$ CEP. Tracking range of module is approximately $161 \mathrm{dBm}$. It utilizes a 28 constellation of $24 / 32$ active satellites in Earth orbit that transmit an accurate microwave signal And enable GPS receiver. A GPS receiver requires at least three or four satellites to calculate the distance and direction as shown in Figure given below and figure out its two dimensions, that is latitude and longitude or three dimensions that is latitude, longitude, and altitude positions. It consists of satellites, control and monitor stations, and receivers. GPS receivers take information transmitted from the satellites and uses triangulation to calculate a user's exact location [5].

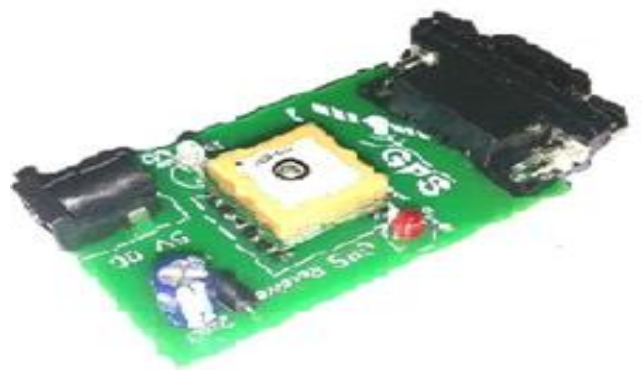

Fig 7: GPS module

\section{SOFTWARE DESCRIPTION}

The software mikroC Pro is used for the programming of PIC18F452 microcontrollers. mikroC is used to develop embedded system applications. PIC microcontroller is used extensively in the world. C language is used mostly to program the microcontrollers, since it becomes has benefits to program the microcontroller in $\mathrm{C}$, because of large libraries available in the $\mathrm{C}$ language. Various features made available to the user by the mikroC includes ANSI compliant compiler, very advanced Integrated Development Environment, comprehensive documentation, large libraries for hardware, and enriched set of examples.

To design PCB a software OrCAD is used. Once testing of all the possible modules has been completed using a general purpose PCB, the final hardware for the product was it invdeveloped. To ensure a reliable system, professional prototype was developed. It involves mainly two steps schematic design and PCB layout. The first step in process of PCB designing is Schematic design. The schematic gives a visual demonstration of the connections of the electronic components used. Schematic is made very easily by using OrCAD software in just few steps. There is just a need to select component, place them and then connect them with wires. Next step is to layout the print circuit board. The net list contains the information about what are components in circuit and how are they connected with each other so there is no need to re enter the information manually. Net list also includes information about component sizes and the packages for use in creating the PCB artwork. 


\section{TEST RESULT AND DISCUSSION}

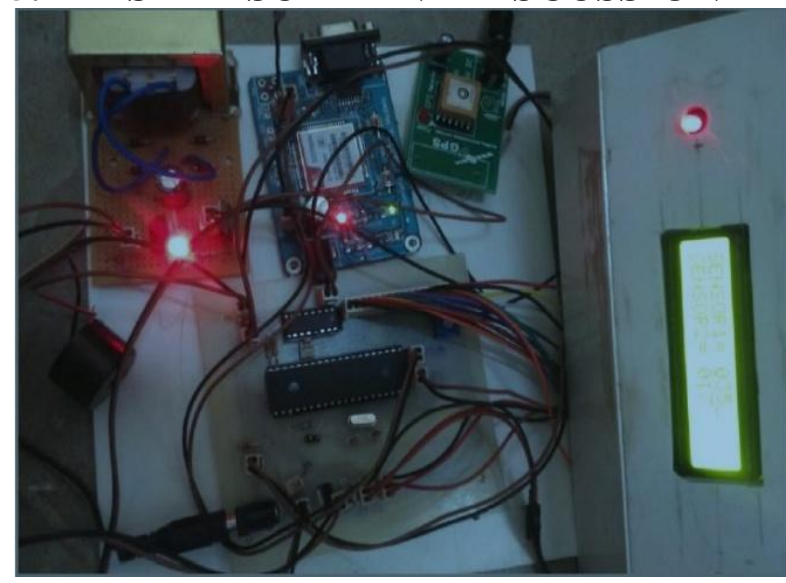

Fig 8: Prototype of system

The prototype designed shown all the components used and the working of system. when the level of fume increased up to the typical level then the system starts operating as the control system starts and alert the user as LED glows and alarm system starts working. If the level continuously increasing and reaches up to the danger level then the window of the car open automatically by the motor and a message is sent if the level is high after the ventilation is provided which is having the current location of the vehicle which acquired by the GPS and a message is sent using GSM.

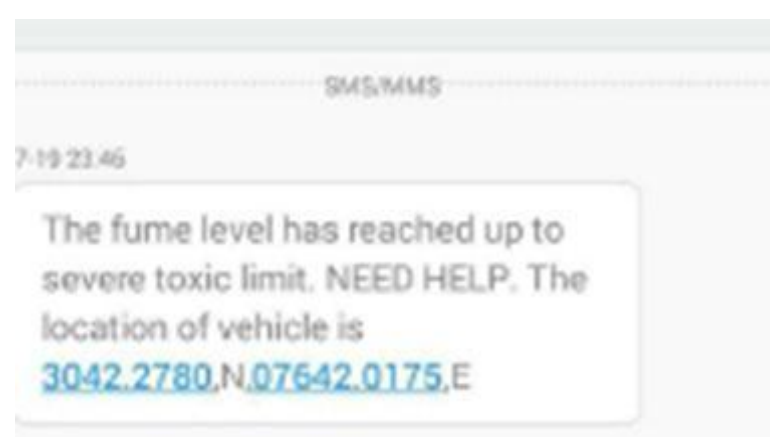

Fig 9: Message sent using GSM and GPS

The performance of prototype was checked in different environmental conditions by generating same amount of carbon monoxide gas at different time slots. The change in the concentration of carbon monoxide gas at different time slot is shown in table 2 .

Table 2 Concentration of $\mathrm{CO}$ at different environmental condition at different time

\begin{tabular}{|c|c|c|c|c|}
\hline S.no & $\begin{array}{c}\text { Time } \\
(\mathbf{s})\end{array}$ & $\begin{array}{c}\text { Normal } \\
(\mathbf{p p m})\end{array}$ & $\begin{array}{c}\text { Under } \\
\text { sun } \\
(\mathbf{p p m})\end{array}$ & $\begin{array}{c}\text { Moving } \\
\text { condition } \\
(\mathbf{p p m})\end{array}$ \\
\hline 1 & 15 & 24 & 40 & 21 \\
\hline 2 & 30 & 28 & 44 & 23 \\
\hline 3 & 45 & 29 & 46 & 27 \\
\hline 4 & 60 & 33 & 51 & 28 \\
\hline 5 & 75 & 41 & 57 & 29 \\
\hline 6 & 90 & 46 & 63 & 31 \\
\hline 7 & 105 & 51 & 68 & 36 \\
\hline
\end{tabular}

\begin{tabular}{|c|c|c|c|c|}
\hline 8 & 120 & 57 & 72 & 39 \\
\hline 9 & 135 & 69 & 76 & 44 \\
\hline 10 & 150 & 75 & 89 & 47 \\
\hline 11 & 165 & 79 & 97 & 54 \\
\hline 12 & 180 & 86 & 108 & 58 \\
\hline 13 & 195 & 94 & 119 & 66 \\
\hline 14 & 210 & 99 & 124 & 74 \\
\hline 15 & 225 & 105 & 129 & 76 \\
\hline
\end{tabular}

\section{- Vehicle testing}

In the vehicle test the $\mathrm{CO}$ distribution was measured in the vehicle cabin. Carbon monoxide was brought in between evaporator and heater core. This test allows checking the concentration of $\mathrm{CO}$ in the compartment. For measurement, six concentrations of $\mathrm{CO}$ distributed at different positions in the cabin were detected. The concentration of $\mathrm{CO}$ was measured at different time slots as shown in figure 10 .

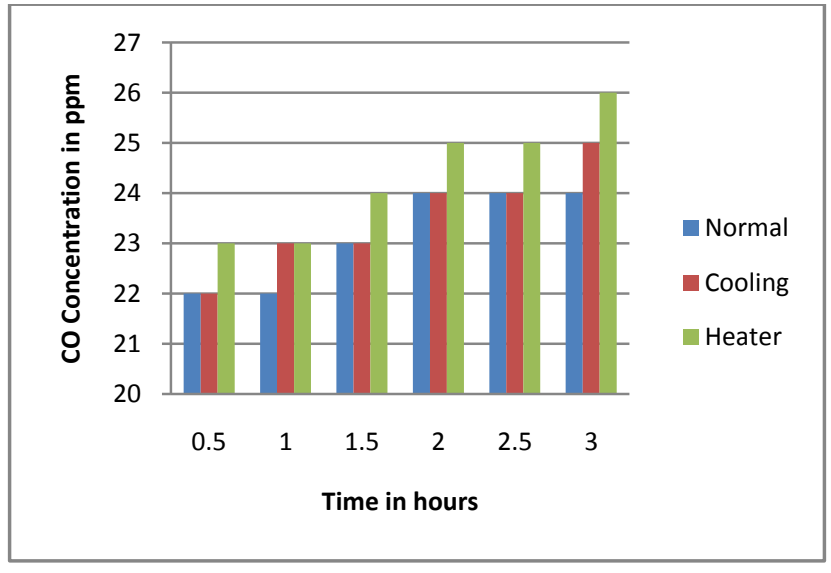

Figure 10: CO concentration at different time slots in the vehicle cabin.

\section{CONCLUSION}

The prototype comprises of a sensing and control unit. In the sensing unit sensor detects the level of carbon monoxide inside the car cabinet. Output of gas sensor is displayed on the LCD screen. Control unit continuously monitors the gas sensor output. If the value increases then LED glows to show the danger level and buzzer sounds which alerts the user about the danger level. If the level continuously rises then motor opens the window so that the ventilation can be provided and the level of fume can reduce and danger level also become lesser. The GSM and GPS module is used to send the message to the related person about the situation and the location of the vehicle. The reference value helps distinguish about the danger level and the normal level of gas. The prototype continuously runs whether the level is high or low it continuously monitors the environment. The prototype is tested and successfully demonstrates the intended operation. In summary, this prototype demonstrates that this idea can be implemented for real time application. 


\section{REFERENCES}

[1] Ma Shuhua, Gu Qiongchan and Lv Jiangtao, "A CO detection system based on double fiber bragg gratings," in proc. Photonics Global Conference, pp.1-3, December 13-16, 2012.

[2] M. Mello, A. de Risi, A. Passaseo, M. Lomascolo and M. De Vittorio, "Carbon-monoxide (CO) Real Time Monitoring in Combustion Engines by an Optical Detection System," Research in Microelectronics and Electronics, pp.433-436, 2006

[3] B. Raic and A. Radovan, "Microcontroller managed module for automatic ventilation of vehicle interior," in Proc. of 37th International Convention on Information and Communication Technology, pp.1622-1627, 26-30 May 2014.

[4] "LCD interfacing tutorial: Introduction tutorial: http://www.8051 projects.net/lcdinterfacing/introduction.php

[5] Michael Carpenter and Dr. Shanker Balasubramaniam, "Interfacing a GPS Receiver with a Microprocessor via UART Application note," November 13, 2009.
[6] T. Takada "New method for gas identification using a single semiconductor sensor," Research in sensors and actuators B: chemical science direct Journal, vol. 66, pp.22-24, 2000.

[7] Campus Components. Available online at: http://www.campuscomponent.com/wirelessmodules/cirocomm-gps-chips-36,Jan 19,2010.

[8] Michel Mouly and Marie-Bernadette Pautet, The Gsm System for Mobile Communications. Telecom pub, 1992.

[9] N. Nandhini, G. Vinoth Chakkaravarthy and G. Deepa Priya, "Talking Assistance about Location Finding," International Journal of Innovative Research in Science, Engineering and Technology, Vol. 3, pp. 9646-9651, February 2014

[10] Charathip Chunkul, Khanchai Tunlasakun, and Ratchadawan Nimnual, "PIC Implementation of Carbon Monoxide Alarm for Indoor Parking Car," in Proc International Conference on Control Automation and Systems, vol. 2 pp. 850 - 854 October 14-17, 2008. 